\title{
MM-Wave Phased Array Quasi-Yagi Antenna for the Upcoming 5G Cellular Communications
}

\author{
Naser Ojaroudi Parchin ${ }^{1, *(1)}$, Mohammad Alibakhshikenari ${ }^{2}\left(\mathbb{D}\right.$, Haleh Jahanbakhsh Basherlou ${ }^{3}(\mathbb{D}$, \\ Raed A. Abd-Alhameed ${ }^{1}\left(\mathbb{D}\right.$, Jonathan Rodriguez ${ }^{4,5}$ and Ernesto Limiti ${ }^{2} \mathbb{C}$ \\ 1 Faculty of Engineering and Informatics, School of Electrical Engineering and Computer Science, University \\ of Bradford, Bradford BD7 1DP, UK; R.A.A.Abd@bradford.ac.uk \\ 2 Electronic Engineering Department, University of Rome Tor Vergata, Via del Politecnico 1, 00133 Rome, Italy; \\ Alibakhshikenari@ing.uniroma2.it (M.A.); Limiti@ing.uniroma2.it (E.L.) \\ 3 Bradford College, West Yorkshire, Bradford BD7 1AY, UK; Hale.Jahanbakhsh@gmail.com \\ 4 Instituto de Telecomunicações-Aveiro, Campus Universitário de Santiago, 3810-193 Aveiro, Portugal; \\ Jonathan@av.it.pt \\ 5 Mobile and Satellite Communications Research Group, University of South Wales, Treforest CF37 1DL, UK \\ * Correspondence: N.OjaroudiParchin@Bradford.ac.uk; Tel.: +44-734-143-6156
}

Received: 21 January 2019; Accepted: 5 March 2019; Published: 8 March 2019

\begin{abstract}
The focus of this manuscript was to propose a new phased array antenna design for the fifth generation (5G) mobile platforms. Eight elements of compact Quasi-Yagi antennas were placed on the top portion of smartphone printed circuits board (PCB) to form a beam-steerable phased array design. The $-10 \mathrm{~dB}$ impedance-bandwidth of proposed 5G smartphone antenna spans from $25 \mathrm{GHz}$ to $27 \mathrm{GHz}$ providing $2 \mathrm{GHz}$ bandwidth with less than $-16 \mathrm{~dB}$ mutual coupling function. A coax-to-microstripline with a truncated crown of vias around the coaxial cable was used as a feeding mechanism for each radiation element. An Arlon Ad 350 substance with properties of $\varepsilon=3.5, \delta=0.003$, and $\mathrm{h}=0.8 \mathrm{~mm}$ was chosen as the antenna substrate. The proposed phased array antenna provides wide-angle scanning of $0^{\circ} \sim 75^{\circ}$ with more than $10 \mathrm{~dB}$ realized gain levels. For the scanning angle of $0^{\circ} \sim 60^{\circ}$, the antenna array provides more than $90 \%(-0.5 \mathrm{~dB})$ radiation and total efficiencies. In addition, the specific absorption rate (SAR) function and radiation performance of the design in the presence of the user-hand/user-hand have been studied. The results validate the feasibility of the proposed design for use in the $5 \mathrm{G}$ handheld devices. Furthermore, using the presented Quasi-Yagi elements, the radiation properties of $2 \times 2,4 \times 4$, and $8 \times 8$ planar arrays were studied and more than $8.3,13.5$, and $19.3 \mathrm{dBi}$ directivities have been achieved for the designed planar arrays. The results show that the designed arrays (linear \& planar) satisfy the general requirements for use in $5 \mathrm{G}$ platforms.
\end{abstract}

Keywords: 5G systems; end-fire radiation beam; cellular communications; phased array; mm-Wave applications; Quasi-Yagi antenna

\section{Introduction}

One of the issues expected for the fifth generation (5G) cellular communications is the move to the millimeter-Wave (mm-Wave) bands [1], which has received great attention from many researchers and industry. There has been a lot of reported research efforts on mm-Wave-based 5G communication systems [2]. The $5 \mathrm{G} \mathrm{mm-Wave} \mathrm{communication} \mathrm{technology} \mathrm{can} \mathrm{handle} \mathrm{over} \mathrm{a} \mathrm{thousand} \mathrm{times} \mathrm{more}$ mobile traffic than previous mobile communication networks such as 4G LTE [3]. Moving to mm-Wave bands would bring new challenges and certainly will require careful consideration on the antenna design for the $5 \mathrm{G}$ handheld devices. The $26 \mathrm{GHz}$ is one of the promising frequency bands for $\mathrm{mm}$-Wave $5 \mathrm{G}$ communications, which has been proposed by Ofcom, UK [4]. Compact antennas arranged as 
an array can be employed at different portions of a smartphone PCB to form linear phased arrays with high gain and directional radiation beams. Different from conventional antennas, such as patch, slot, or monopole antennas, to cover the required coverage-space of 5G cellular communications, the end-fire antennas, such as horn, Yagi-Uda, Quasi-Yagi, LTSA, Dipole, and Vivaldi, are more suitable [5-11]. Among them, the Quasi-Yagi antenna is a promising candidate due to its attractive features, such as compact size, high gain, and easy integration.

The Quasi-Yagi antenna is a widely used antenna with high gain end-fire radiation patterns. Its configuration consists of a reflector and a driven element along with one or multiple directors. It can provide excellent directional propagation at the higher frequencies and could be used for radar, detecting, and phased array applications [12]. This study investigates the configuration and characteristics of the $\mathrm{mm}$-Wave phased array antenna with Quasi-Yagi radiation elements for use in 5G cellular platforms. Eight Quasi-Yagi antenna elements are placed on the top side of the smartphone PCB and fed by 50-Ohm coax-to-microstriplines [13]. Each element is composed of driving dipole arms with a pair of director elements on the top layer of the substrate. For the handheld devices, the measurement of end-fire microstrip-fed antennas (such as Bow-tie, Dipole, Yagi, Vivaldi, etc.) can only be performed using cable due to the ground plane of smartphone PCBs [14-17]. We propose here a new coax-to-microstripline feeding technique to achieve a better transition. The designed 5G smartphone antenna has $2 \mathrm{GHz}$ (below $-10 \mathrm{~dB} \mathrm{~S}_{\mathrm{nn}}$ ) in the frequency range of 25 to $27 \mathrm{GHz}$. Good radiation properties in terms of the antenna's fundamental properties have been obtained for the antenna. In addition, the designed 5G antenna provides sufficient SAR levels with good behavior in the vicinity of the user's hand. The planar phased array configurations of the Quasi-Yagi antenna with $2 \times 2,4 \times 4$, and $8 \times 8$ radiation elements were also designed and high directivity radiation beams have been obtained. Simulations were carried out using computer simulation technology (CST) software [18].

\section{Quasi-Yagi Antenna}

In recent years, printed Quasi-Yagi antennas have been exhaustively analyzed and experimentally researched. According to the driven element form, the existing Quasi-Yagi antenna can be divided into a microstrip patch, double-sided dipole, or single-sided dipole form [19]. A schematic of the designed Quasi-Yagi antenna is displayed in Figure 1. The antenna was designed on a $0.8 \mathrm{~mm}$ Arlon Ad 350 $(\varepsilon=3.5$ and $\delta=0.003)$ dielectric to operate at $26 \mathrm{GHz}$.

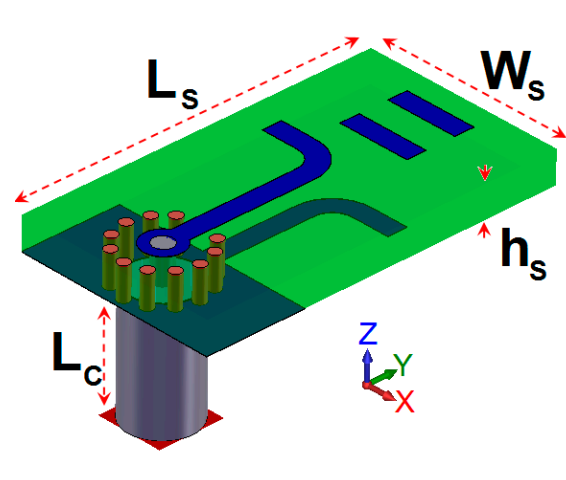

(a)

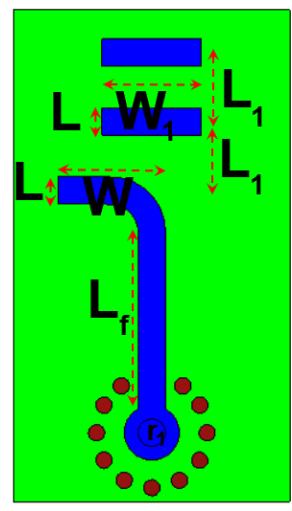

(b)

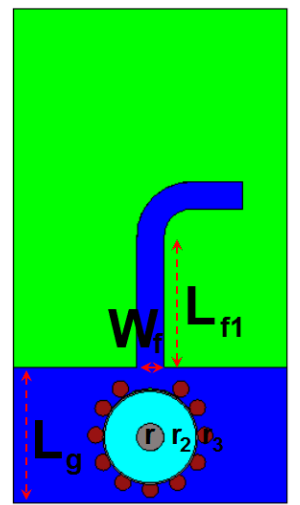

(c)

Figure 1. Quasi-Yagi antenna schematic; (a) transparent view, (b) top, and (c) bottom layers.

As shown in Figure 1, the antenna is fed by a coax-to-microstripline. The feeding point of the coaxial cable has been surrounded by a cylindrical metal vias. It is seen that the inner connector of the probe is connected to the feed line of the Yagi's arm while the outer connector has been connected to the ground plane. The final dimensions of the proposed Quasi-Yagi antenna and its linear array parameters are specified in Table 1. Figure 2 depicts the frequency response $\left(\mathrm{S}_{11}\right)$ of the Quasi-Yagi 
antenna. For comparison purposes, the antenna element was also designed using Ansoft high frequency structure simulator (HFSS) software [20], and its $S_{11}$ result has been added to Figure 2. As shown, more than $2 \mathrm{GHz}$ bandwidth with good impedance-matching function in the frequency range of 25 to $27 \mathrm{GHz}$ has been achieved.

Table 1. Parameter values of the Quasi-Yagi antenna and its array design.

\begin{tabular}{cccccc}
\hline Parameter & Value $(\mathbf{m m})$ & Parameter & Value $(\mathbf{m m})$ & Parameter & Value $(\mathbf{m m})$ \\
\hline $\mathrm{W}_{\text {sub }}$ & 60 & $\mathrm{~L}_{\text {sub }}$ & 120 & $\mathrm{~h}_{\mathrm{S}}$ & 0.8 \\
$\mathrm{~W}_{\mathrm{S}}$ & 4.5 & $\mathrm{~L}_{\mathrm{g}}$ & 2.5 & $\mathrm{~W}_{\mathrm{f}}$ & 0.5 \\
$\mathrm{~L}_{\mathrm{f}}$ & 3.15 & $\mathrm{~L}_{\mathrm{C}}$ & 3 & $\mathrm{~W}$ & 1.95 \\
$\mathrm{~L}$ & 0.1 & $\mathrm{~W}_{\mathrm{a}}$ & 40 & $\mathrm{~L}_{\mathrm{S}}=\mathrm{L}_{\mathrm{a}}$ & 9 \\
$\mathrm{~W}_{1}$ & 1.8 & $\mathrm{~L}_{1}$ & 1.25 & $\mathrm{r}_{1}$ & 0.5 \\
$\mathrm{r}_{2}$ & 0.87 & $\mathrm{r}_{3}$ & 0.15 & $\mathrm{R}$ & 0.25 \\
\hline
\end{tabular}

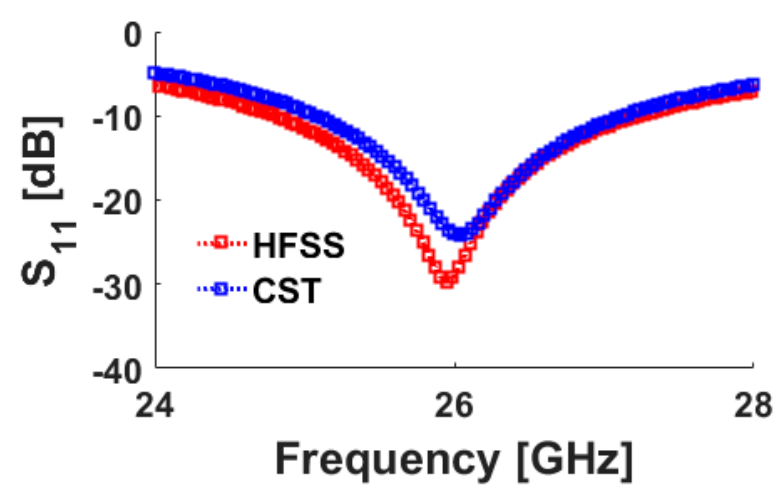

Figure 2. $S_{11}$ function of the antenna.

The operation frequency of the Quasi-Yagi antenna can be controlled by changing the size of the critical parameters. Based on the obtained results in Figure $3 a, b$, the resonance frequency of the antenna can be tuned by changing the values of $\mathrm{W}$ (width of antenna arm) and $\mathrm{Lg}_{\mathrm{g}}$ (length of the ground plane). Furthermore, as evident from Figure $3 c$, the length of the directors $\left(W_{1}\right)$ has a significant impact on the matching function of the designed Quasi-Yagi antenna. Figure 4a represents the simulated surface current densities of the Quasi-Yagi antenna at its resonance frequency (26 GHz): the current flows are opposite around the antenna driving arms and directors. The radiation pattern of the antenna at $26 \mathrm{GHz}$ is displayed in Figure $4 \mathrm{~b}$ : the Quasi-Yagi antenna exhibits an end-fire radiation pattern and provides $4.4 \mathrm{~dB}$ realized gain level at its frequency resonance. The realized gain is the power gain of the antenna including mismatch losses [21]. It should be noted that the directors of the antenna improve the radiation behavior and impedance-matching of the designed Quasi-Yagi antenna [22]. Figure 5 illustrates the Quasi-Yagi radiation and total efficiencies versus its impedance bandwidth. It can be observed that high-efficiency characteristics (radiation efficiency $>95 \%$ and total efficiency $>85 \%$ ) have been achieved over the antenna operation bandwidth.

Using the directors, the antenna can provide a well-defined and more directional end-fire radiation pattern. It can also improve the gain level of the antenna at the operation frequency. Figure 6 illustrates a comparison of the antenna radiation behavior with/without the directors. As seen in Figure 6a, the antenna with directors can provide a good radiation pattern with higher gain and efficiency properties at $26 \mathrm{GHz}$ compared with the antenna without the directors. In addition, as illustrated in Figure $6 \mathrm{~b}$, using the directors, the realized gain function of the antenna over the operation band has been increased significantly. 


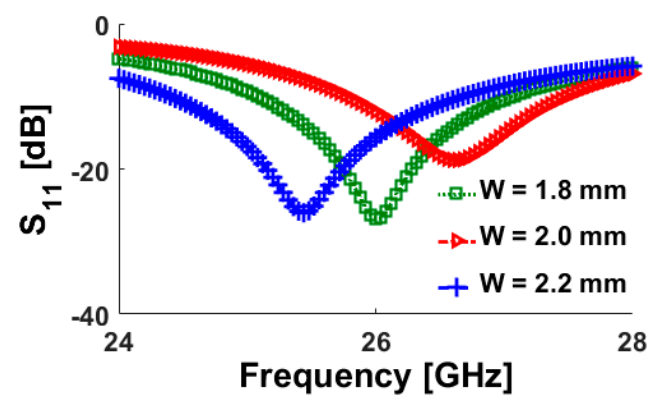

(a)

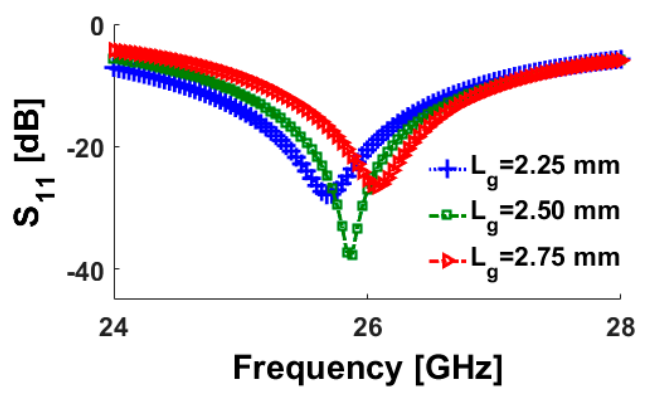

(b)

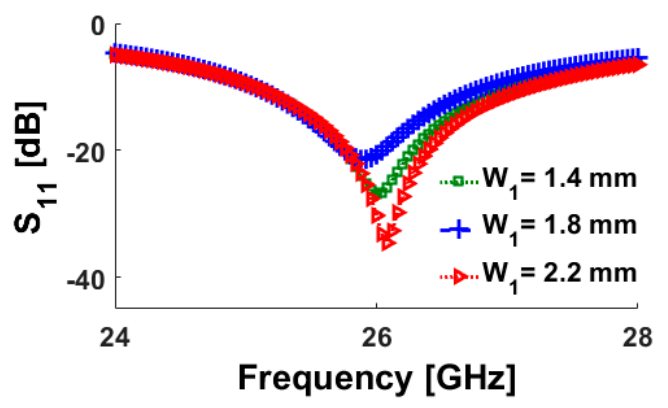

(c)

Figure 3. $S_{11}$ results of the Quasi-Yagi antenna for different values of (a) W (width of the antenna arm), (b) $\mathrm{L}_{\mathrm{g}}$ (length of the ground plane), and (c) $\mathrm{W}_{1}$ (length of the directors).

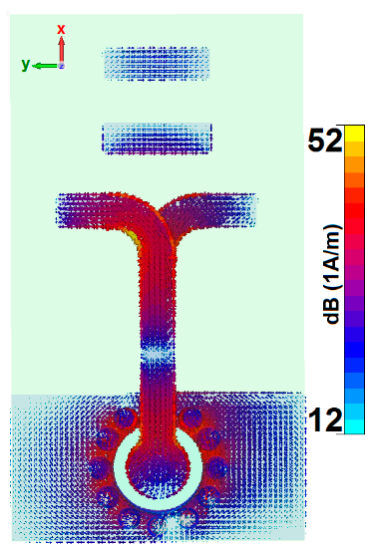

(a)

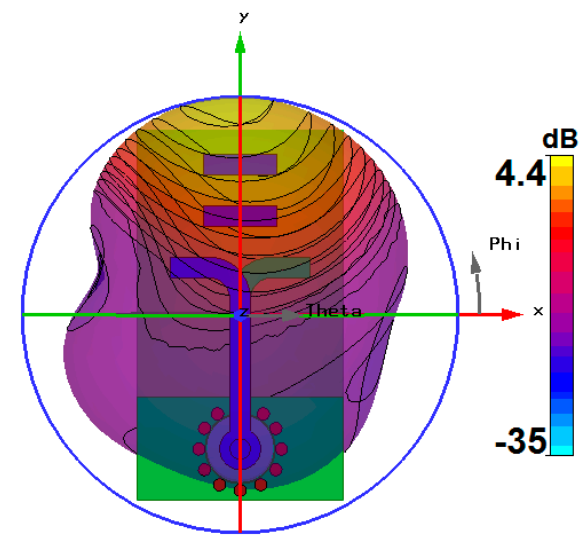

(b)

Figure 4. (a) Current densities and (b) 3D radiation pattern at $26 \mathrm{GHz}$.

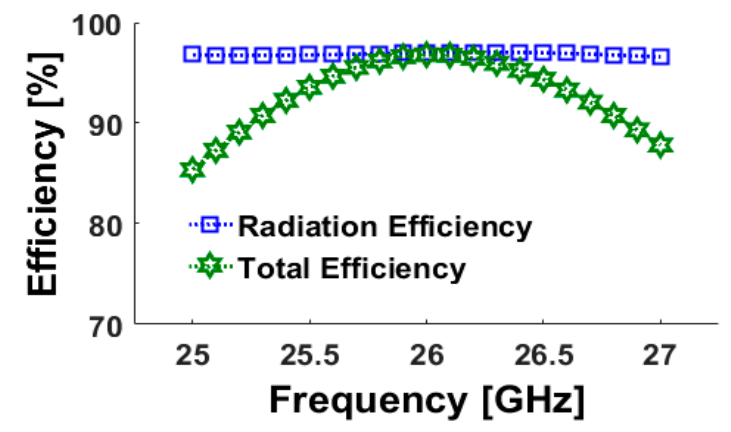

Figure 5. Simulated efficiencies. 


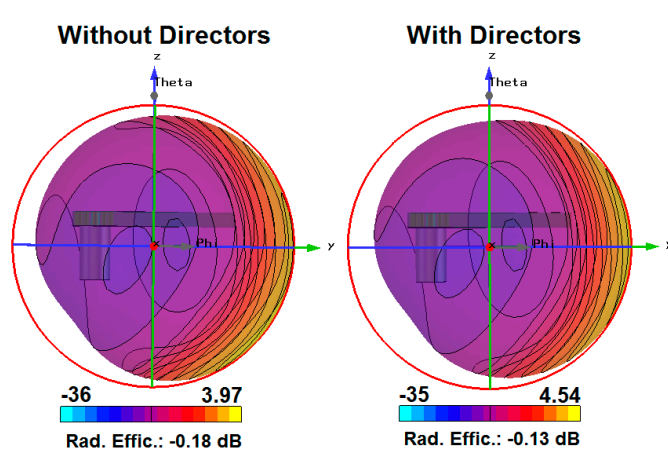

(a)

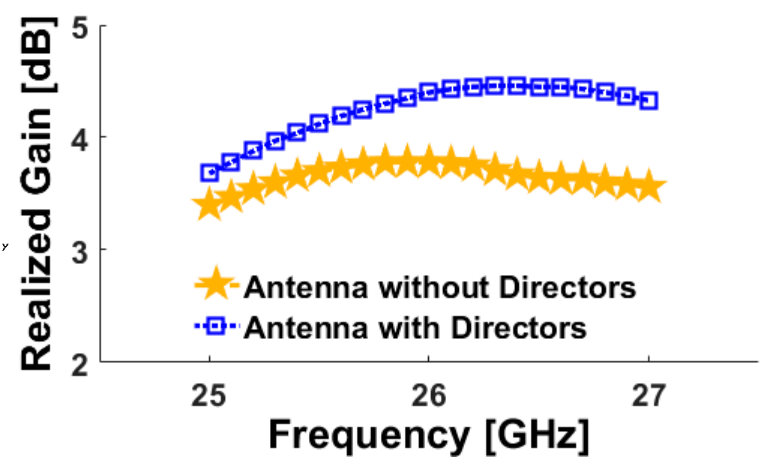

(b)

Figure 6. Comparison of the antenna performance with/without directors: (a) radiation pattern and (b) gain.

In order to investigate the performance of the Quasi-Yagi antenna as a transmitter/receiver in a mobile terminal, the fidelity factor of the proposed design has been calculated. Two identical antennas as transmitter $\left(T_{X}\right)$ and receiver $\left(R_{X}\right)$ are placed with $100 \mathrm{~mm}$ shift of their center points. The antenna orientations are arranged in side by side and face to face configurations. Figure 7 illustrates the transmitted signal in free space and received pulses. The calculated fidelity factor of the antenna for the face to face and side by side configurations are equal to 0.90 and 0.98 , respectively. The achieved fidelity results confirm that the designed Quasi-Yagi antenna imposes negligible effects on the transmitted pulses [23].

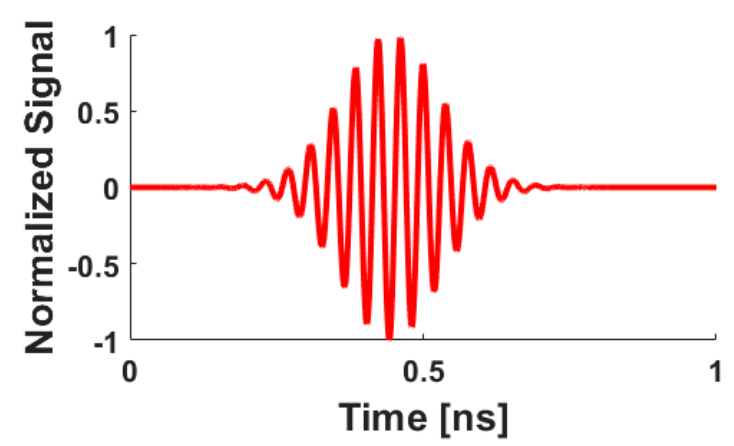

(a)

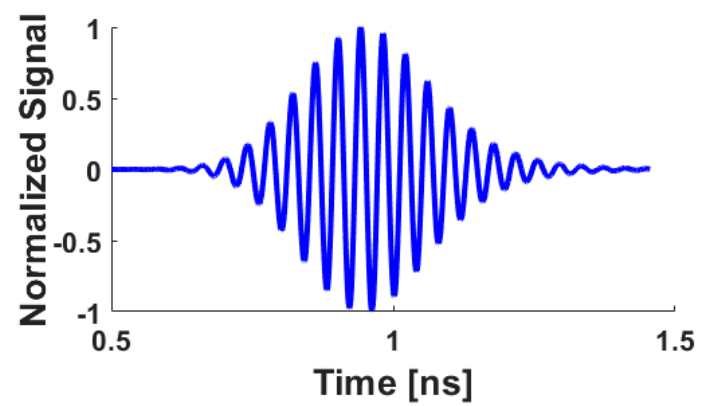

(b)

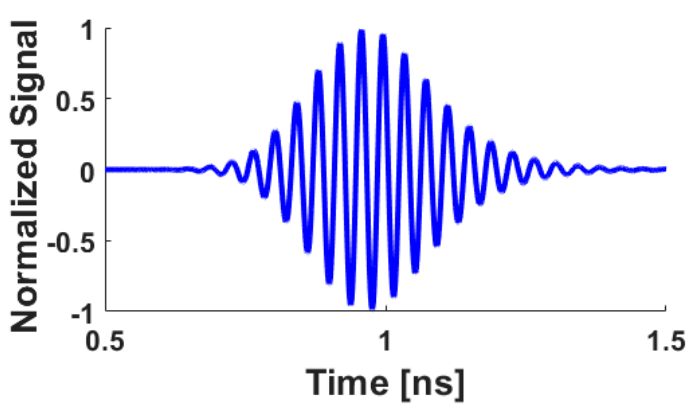

(c)

Figure 7. (a) Transmitted signal in free space and received pulses for (b) side by side and (c) face to face.

\section{The Phased Array 5G Smartphone Antenna}

Transparent and top views of a linear phased array design with the Quasi-Yagi antenna elements are shown in Figure 8. It has a low profile of $\mathrm{W}_{\mathrm{a}} \times \mathrm{L}_{\mathrm{a}}=9 \times 40 \mathrm{~mm}^{2}$. The antenna elements are arranged with a distance of $d=5 \mathrm{~mm}$. As it can be observed, it is composed of eight $26 \mathrm{GHz}$ Quasi-Yagi antenna elements and could be used on the top/bottom portions of the smartphone PCB to form two 
sets of linear phased arrays [24,25]. Figure 9 shows a phased array system architecture with a feeding network that could be used for the linear phased array Quasi-Yagi antenna and can be demonstrated using phase shifters for beam switching. Various techniques of feeding network design such as parallel and series can be employed for this purpose.

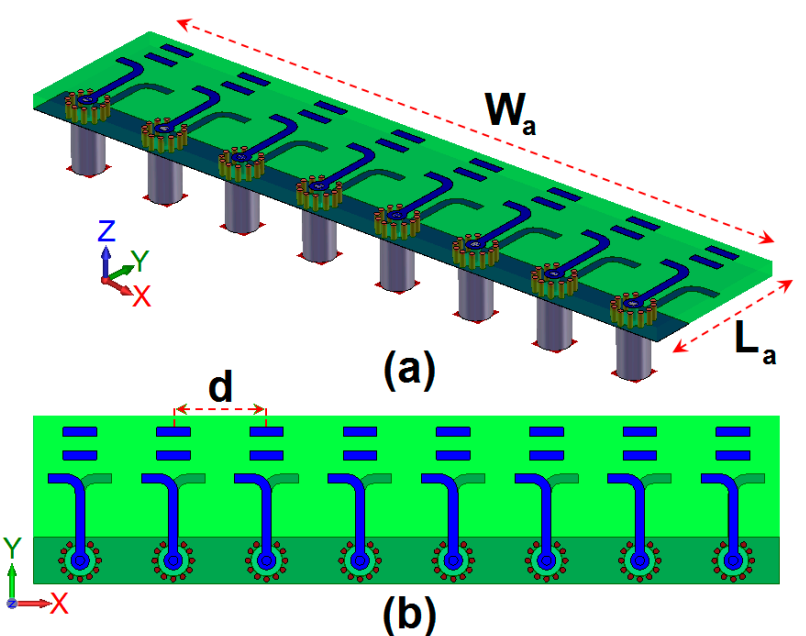

Figure 8. (a) 3D transparent and (b) top views of the linear phased array antenna.

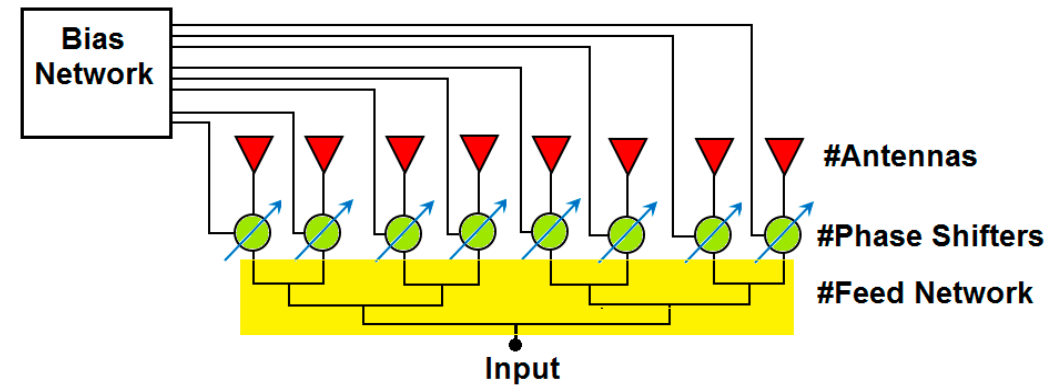

Figure 9. Phased array system architecture.

In array designing, the spacing between the adjacent elements must be determined carefully due to its impact on the fundamental characteristics of the array [26]. The properties of the Quasi-Yagi array with various distances of the adjacent radiation elements have been studied in Figure 10. Its various configurations are illustrated in Figure 10a. Mutual coupling characteristics of the middle element of the array $\left(\mathrm{S}_{21}\right)$ for various distances are plotted in Figure 10b. As seen, for different values of $d$ (3.5 to $6.5 \mathrm{~mm}$ ), the mutual coupling function varies from -8 to $-20 \mathrm{~dB}$. Figure 10c illustrates the 3D radiation beams of the Quasi-Yagi antenna array with directivity values for different values of $d$ at $0^{\circ}$ angle. As seen, by increasing the distance between the elements, the antenna radiation characteristics can be improved. However, on the other hand, the scanning angle of the array will be limited significantly. So, a trade-off between wide scanning and high radiation characteristics is needed.

Figure 11 illustrates the proposed $\mathrm{mm}$-Wave phased array 5G smartphone antenna. The antenna has an overall dimension of $\mathrm{W}_{\text {sub }} \times \mathrm{L}_{\mathrm{sub}}=60 \times 120 \times 0.8 \mathrm{~mm}^{3}$ and has been constructed on the Arlon Ad 350 dielectric. The designed linear array is placed on the top side of the PCB to provide beam-steering function at different scanning angles. In order to acquire a wide-scanning function ( 0 to \pm 75 degree), the antenna elements are placed with a bit less than $\lambda / 2(\mathrm{~d}=5 \mathrm{~mm})$ distance. Another set of the design could be deployed at the bottom side of the smartphone PCB. The $S$ parameter results of the Quasi-Yagi 5G antenna array are shown in Figure 12: Good impedance-matching and same bandwidth of the single element ( 25 to $27 \mathrm{GHz}$ ) have been achieved for the proposed structure. In addition, a low mutual coupling function between the adjacent antenna elements has been achieved. 


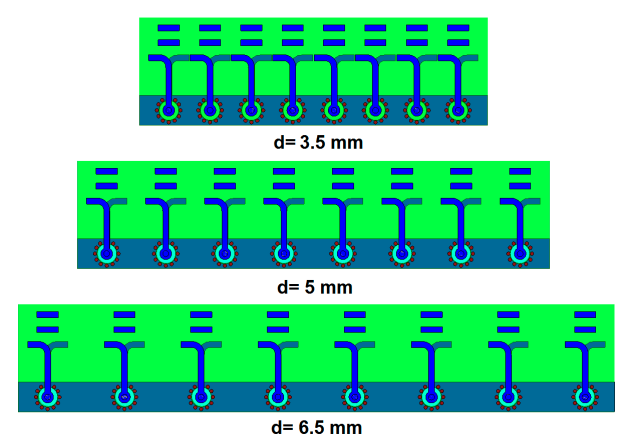

(a)

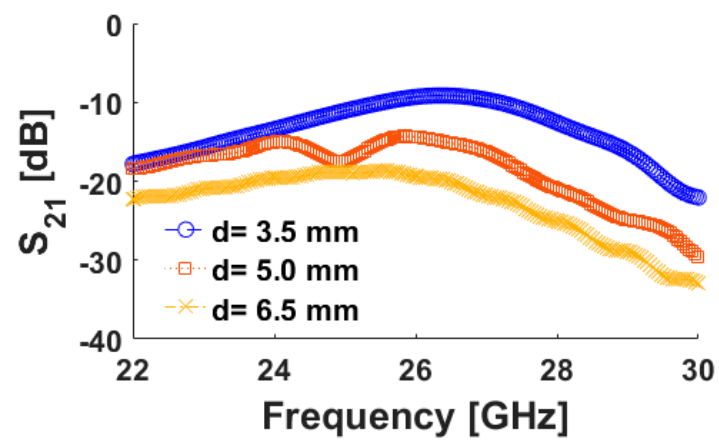

(b)

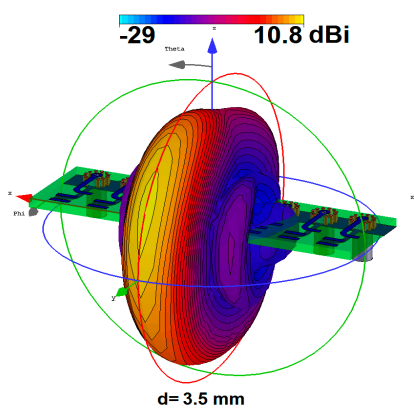

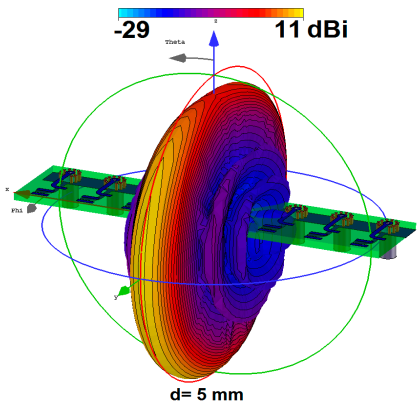

(c)

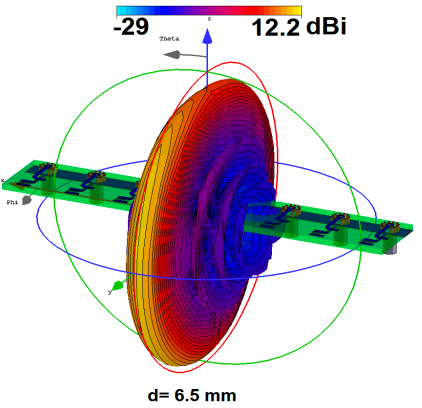

$\mathrm{d}=6.5 \mathrm{~mm}$

Figure 10. (a) Configurations, (b) $\mathrm{S}_{21}$, and (c) radiation beams of the linear array for different vales of $d$.

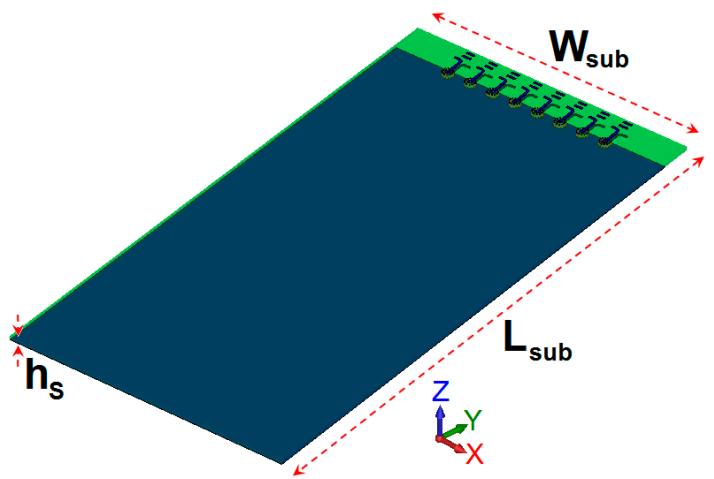

(a)

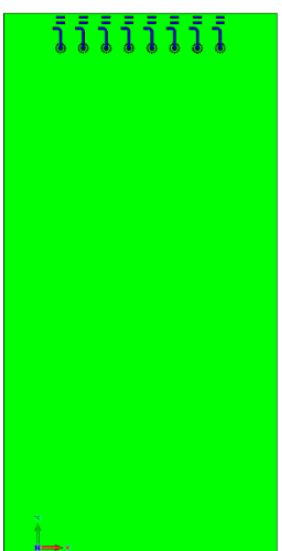

(b)

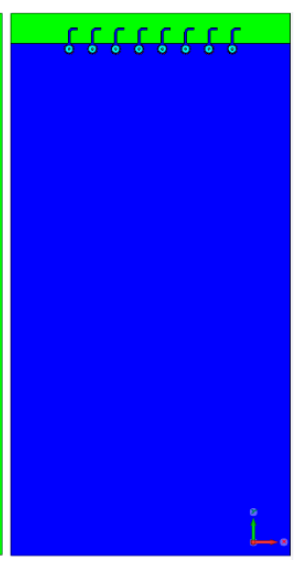

(c)

Figure 11. The mm-Wave smartphone antenna, (a) transparent, (b) top, and (c) bottom views. 


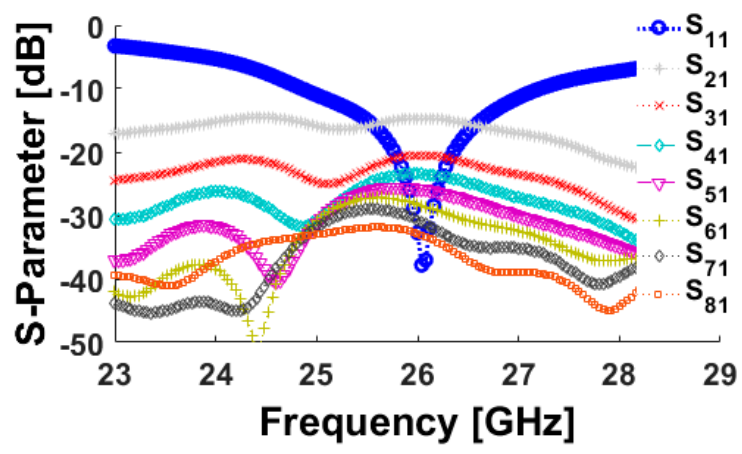

Figure 12. $S$ parameter results of the $\mathrm{mm}$-Wave $5 \mathrm{G}$ smartphone antenna.

The simulated surface current densities at $26 \mathrm{GHz}$ for the phased array 5G smartphone antenna has been illustrated in Figure 13. It can be seen that the current flows are distributed around the radiation arms of the Quasi-Yagi antenna elements on the top side of the smartphone PCB. Each single-element Quasi-Yagi radiator has a high current density and appears very active at the resonance frequency $(26 \mathrm{GHz})[27]$.

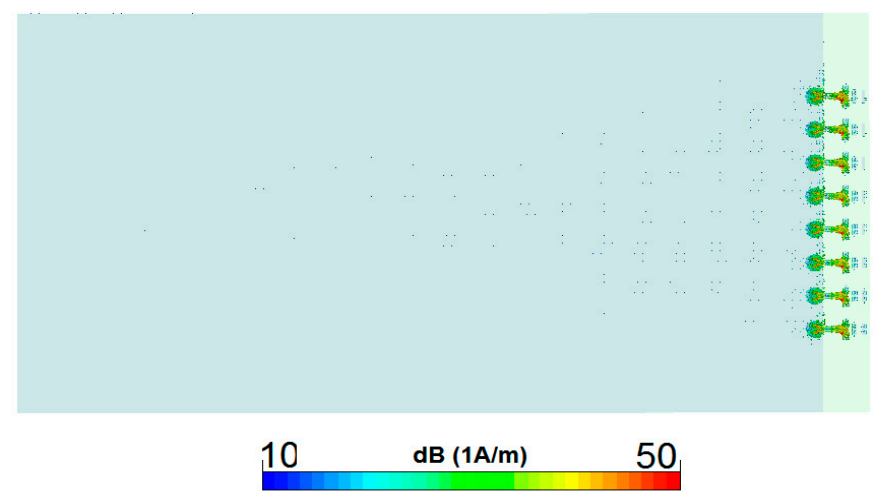

Figure 13. The current distribution at $26 \mathrm{GHz}$.

In order to demonstrate that the ground plane (GND) of the smartphone PCB has an insignificant effect on the radiation behavior of the phased array Quasi-Yagi antenna, the radiation performances of the 5G smartphone antenna with different lengths of the PCB ground plane have been investigated in Figure 14. As illustrated, the size of the PCB ground plane does not have a significant impact on the radiation properties of the array, such as the antenna gain and its efficiency. Less than a $0.3 \mathrm{~dB}$ variation has been obtained in the realized gain level of the smartphone antenna array in the main radiation beam $\left(0^{\circ}\right)$ for different lengths of the PCB ground plane.

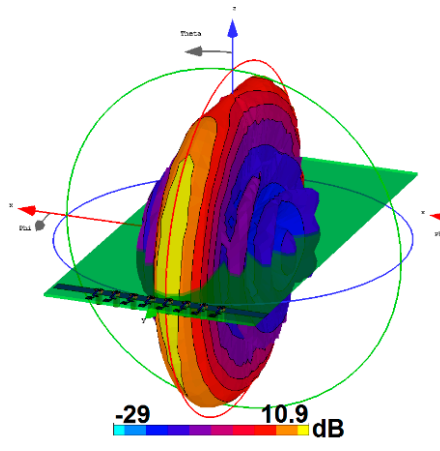

(a)

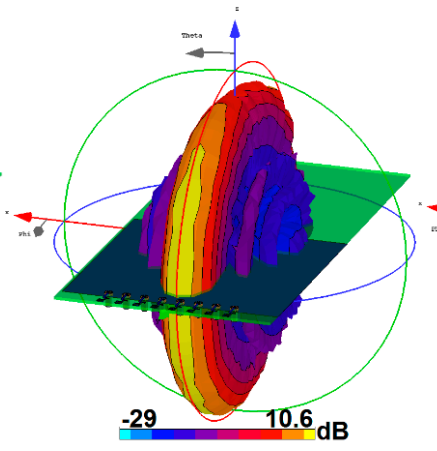

(b)

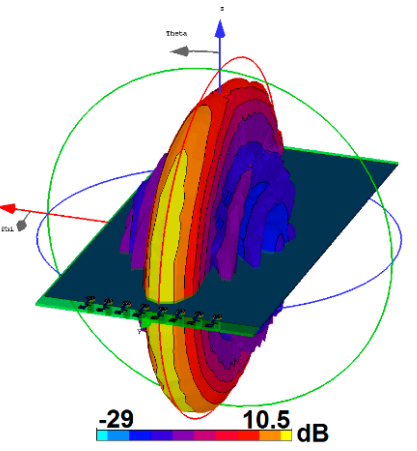

(c)

Figure 14. Radiation beams of the $5 \mathrm{G}$ smartphone antenna at $0^{\circ},(\mathbf{a})$ without GND, (b) half, and (c) full GND. 
3D radiation beams of the 5G smartphone antenna are displayed in Figure 15. The direction and scanning angle of the phased array radiation beams can be assessed by applying the relative amplitudes and phases to the radiators. It can be seen that the presented 5G smartphone antenna has well-defined directional beams with wide-scanning property and high directivity values. In addition, the values of the antenna realized gains at different angles, which are specified in Figure 16a. As shown, the 5G smartphone antenna design exhibits sufficient realized gain levels (more than $10 \mathrm{~dB}$ ) in the scanning range of $0^{\circ}$ to $75^{\circ}$, which makes it suitable for future cellular applications. The $5 \mathrm{G}$ smartphone antenna also provides good efficiencies at different scanning beams. As can be observed from Figure $16 \mathrm{~b}$, more than $-0.5 \mathrm{~dB}(90 \%)$ efficiencies have been obtained in the scanning range of 0-60 degrees. Furthermore, a high directivity characteristic (11-12.5 dBi) has been achieved for the presented design.

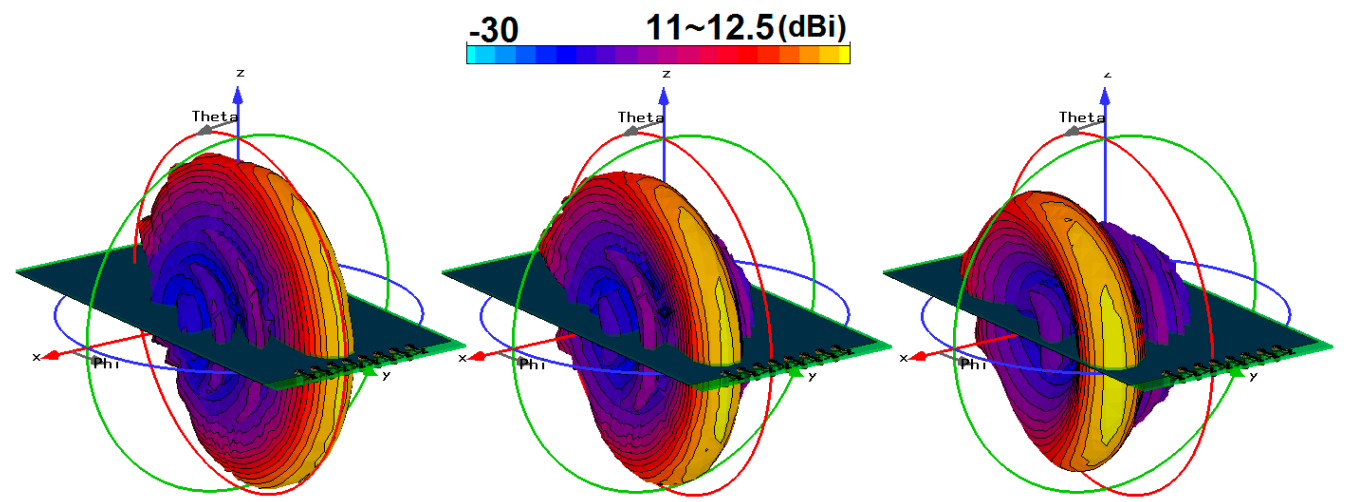

(a)

(b)

(c)

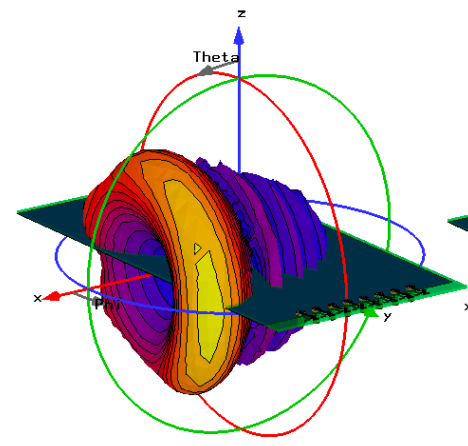

(d)

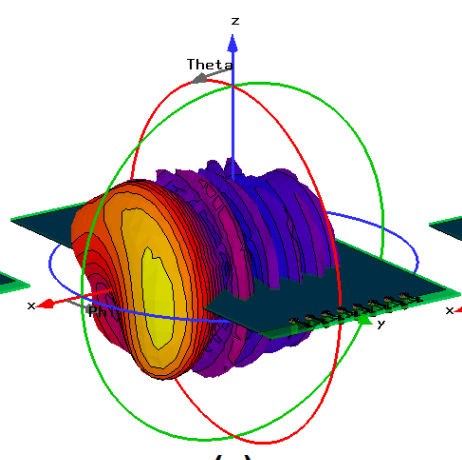

(e)

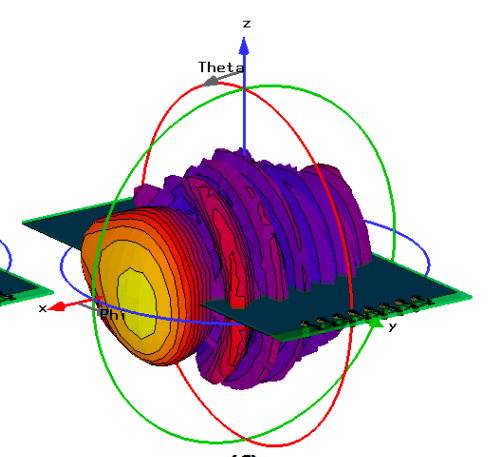

(f)

Figure 15. 3D radiation beams of the $5 \mathrm{G}$ smartphone design at (a) $0^{\circ}$, (b) $15^{\circ}$, (c) $30^{\circ}$, (d) $45^{\circ},(\mathbf{e}) 60^{\circ}$, and (f) $75^{\circ}$.

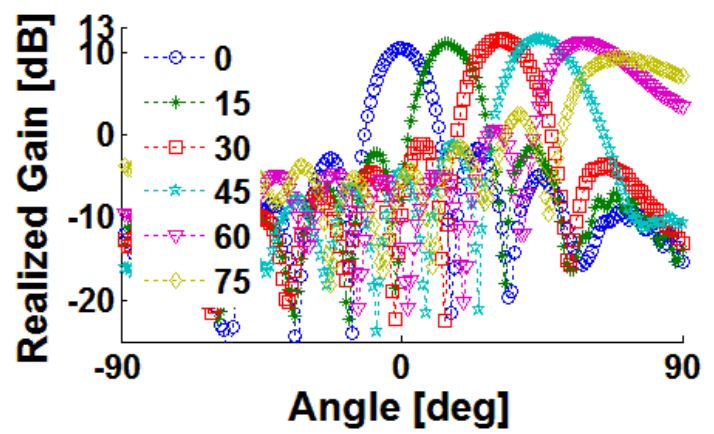

(a)

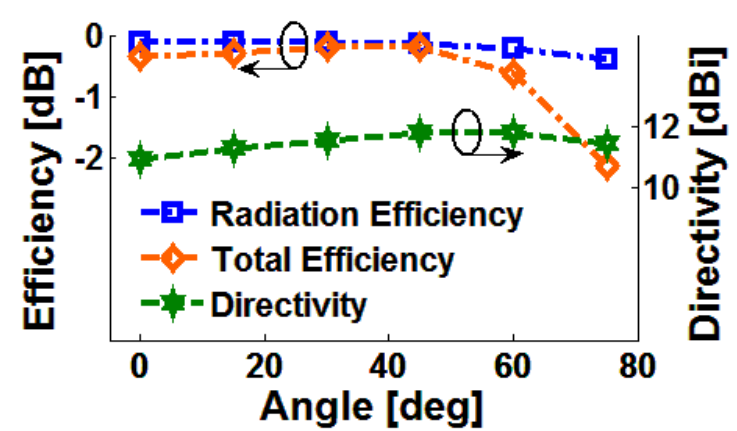

(b)

Figure 16. (a) Realized gain (Cartesian), (b) efficiency and directivity properties at different angles.

Table 2 provides a comparative summary of the fundamental characteristics for the proposed phased array with the recently reported 5G antenna array available in the literature. As can be observed 
from the table, the proposed phased array Quasi-Yagi antenna can support wide scanning angles with high gain and efficiencies. Different from the reported designs, the gain and efficiency characteristics of the design are almost constant over the scanning range of 0-75 degrees. In addition, the antenna elements have more than $18 \mathrm{~dB}$ isolation. Furthermore, the Quasi-Yagi antenna elements are also suitable for use in planar phased array designs for base station applications.

Table 2. Comparison between the proposed and the reported mobile handset antennas.

\begin{tabular}{|c|c|c|c|c|c|c|}
\hline Reference & $\begin{array}{l}\text { Bandwidth } \\
\text { (GHz) }\end{array}$ & $\begin{array}{l}\text { Efficiency } \\
(\%)\end{array}$ & Gain $(\mathrm{dB})$ & $\begin{array}{l}\text { Element } \\
\text { Size }\left(\mathrm{mm}^{2}\right)\end{array}$ & $\begin{array}{l}\text { Isolation } \\
\text { (dB) }\end{array}$ & $\begin{array}{l}\text { Scanning } \\
\text { Range }\end{array}$ \\
\hline [7] & $21-22$ & - & $8-12$ & - & 14 & $0^{\circ} \sim 75^{\circ}$ \\
\hline [8] & $27.5-28.5$ & 70 & $7-11$ & $7 \times 5.5$ & 11 & $0^{\circ} \sim 60^{\circ}$ \\
\hline [9] & $27-29$ & 80 & 8-11 & $5.5 \times 5.5$ & 14 & $0^{\circ} \sim 60^{\circ}$ \\
\hline [10] & $16-18$ & - & $7-11.5$ & $9 \times 9$ & 17 & $0^{\circ} \sim 60^{\circ}$ \\
\hline [11] & $21-23$ & 85 & 9-12 & $12 \times 6$ & 12 & $0^{\circ} \sim 75^{\circ}$ \\
\hline [14] & $27.4-28.8$ & - & $7-11$ & $9 \times 6$ & 16 & $0^{\circ} \sim 60^{\circ}$ \\
\hline [16] & $27-29$ & 80 & $5-9.5$ & $5 \times 4$ & 13 & $0^{\circ} \sim 75^{\circ}$ \\
\hline [17] & $27.5-28.5$ & - & 8-11.5 & - & 15 & $0^{\circ} \sim 60^{\circ}$ \\
\hline Proposed & $25-27$ & 90 & $10.5-12$ & $9 \times 4.5$ & 16 & $0^{\circ} \sim 75^{\circ}$ \\
\hline
\end{tabular}

\section{User-Impact on the Proposed Smartphone Antenna}

One of the human body parts that most frequently touch the smartphone is the user's hands, which usually has a negative impact on the smartphone antenna performance [28-30]. The fundamental radiation characteristics of the 5G smartphone antenna in the presence of the user's hands is studied in this section. The radiation beams of the presented phased array design for a scanning range of 0-75 degrees in the vicinity of the user's hand are represented in Figure 17. As seen, it provides good radiation beams with high directivity values at different angles. Table 3 summarizes the effect of the user's hands on the antenna performance. Compared with the antenna in free space, the overall losses of the smartphone antenna characteristics in terms of antenna gain, radiation, and total efficiencies are less than $3 \mathrm{~dB},-2 \mathrm{~dB}$, and $-2.5 \mathrm{~dB}$, respectively.

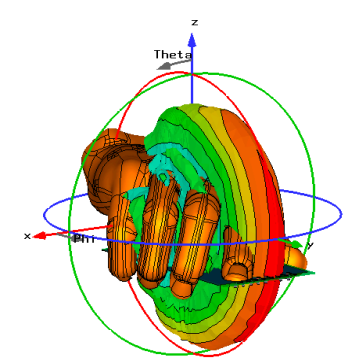

(a)

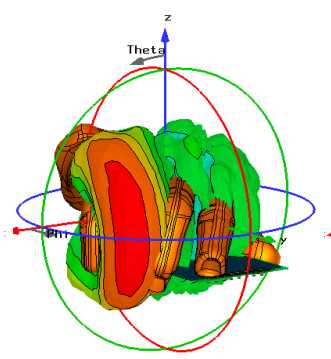

(d)

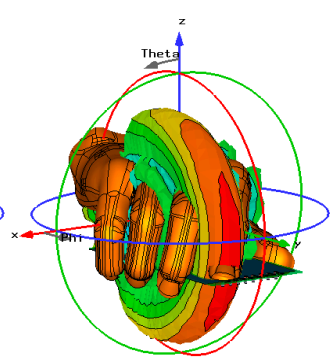

(b)

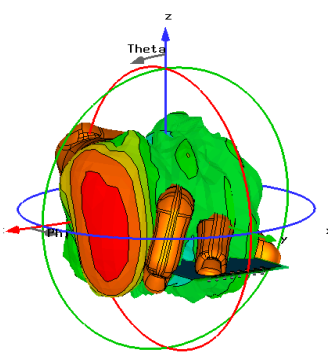

(e)

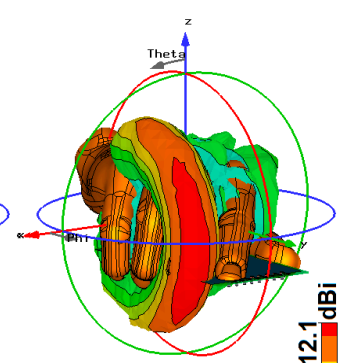

(c)

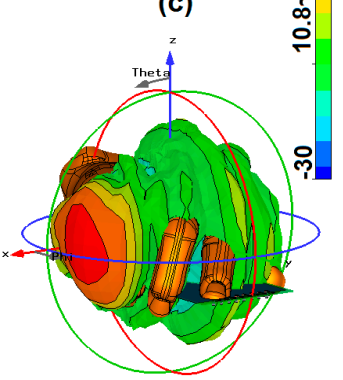

(f)

Figure 17. 3D radiation beams of the $5 \mathrm{G}$ smartphone antenna in the vicinity of the user's hands at (a) $0^{\circ}$, (b) $15^{\circ}$, (c) $30^{\circ}$, (d) $45^{\circ}$, (e) $60^{\circ}$, and (f) $75^{\circ}$. 
Table 3. The properties of the $5 \mathrm{G}$ antenna with the user's hands at different scanning angles.

\begin{tabular}{ccccccc}
\hline Param./Angle & $\mathbf{0}^{\circ}$ & $\mathbf{1 5}^{\circ}$ & $\mathbf{3 0}^{\circ}$ & $\mathbf{4 5}^{\circ}$ & $\mathbf{6 0}^{\circ}$ & $\mathbf{7 5}^{\circ}$ \\
\hline Rad. Effic. (dB) & -1.52 & -1.56 & -1.78 & -2.11 & -2.56 & -2.83 \\
Tot. Effic. (dB) & -2.03 & -2.08 & -2.25 & -2.78 & -2.64 & -4.5 \\
Directivity (dBi) & 11.8 & 12.1 & 12 & 11.92 & 11.6 & 10.8 \\
Rlzd. Gain (dB) & 9.8 & 10 & 9.77 & 9.14 & 8.12 & 6.4 \\
\hline
\end{tabular}

The SAR function of the proposed phased array smartphone antenna is studied in Figure 18. SAR, one of the most critical issues of mobile terminal systems, is a measurement function for absorbed electromagnetic waves by the human body, which should be as low as possible [31,32]. The distance between the 5G smartphone antenna and the user's hand in the z-axis is less than $15 \mathrm{~mm}$. As illustrated in Figure 18, the antenna has sufficient SAR values for different placements. There is a bit of a difference in the SAR values for top and bottom placements of the $5 \mathrm{G}$ antenna, which was predictable due to different distances.

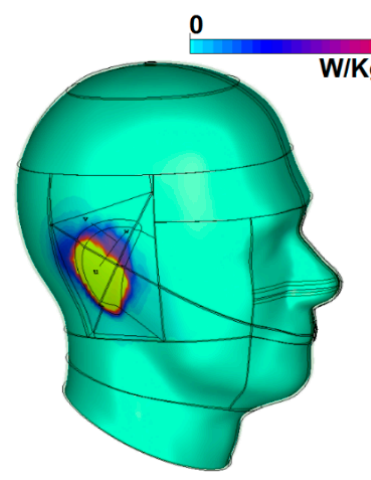

(a)

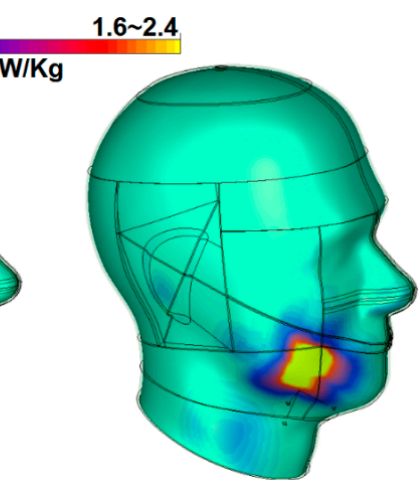

(b)

Figure 18. SAR analysis: (a) top and (b) bottom placements of the 5G smartphone phased array antenna.

\section{Planar Phased Array Design of the $26 \mathrm{GHz}$ Quasi-Yagi Antenna}

The designed $26 \mathrm{GHz}$ Quasi-Yagi antenna can also be used in a planar array form for 5G base station or channel measurement applications [33,34]. Figure 19 illustrates the structures and the directional radiation beams of the designed planar phased array.
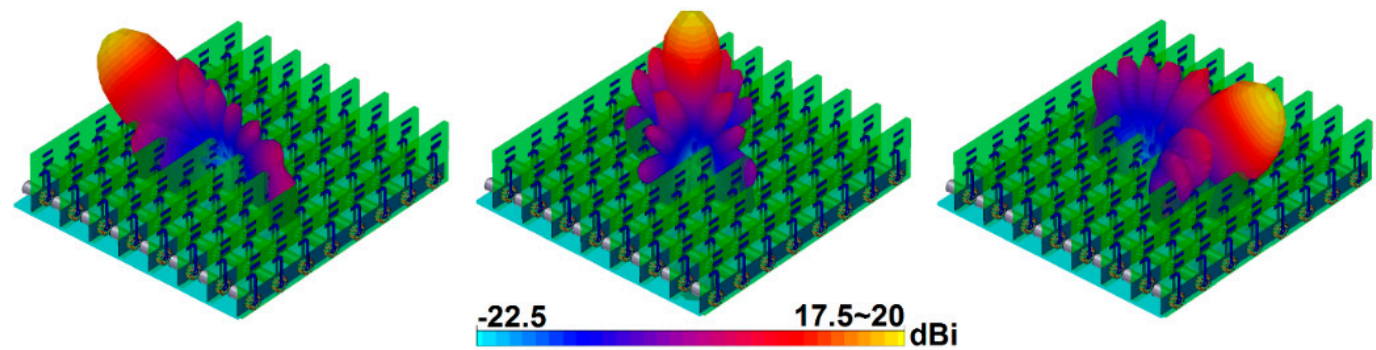

Figure 19. Configuration and 3D beams of the Quasi-Yagi phased array at different angles.

As shown, 64 elements of $26 \mathrm{GHz}$ Quasi-Yagi radiators are employed to form the proposed $8 \times 8$ planar antenna array design. In other words, the planar array has been designed using eight rows of the linear phased array described in Section 3. The proposed planar array antenna works in the same operation band of the single element Quasi-Yagi (frequency range from 25 to $27 \mathrm{GHz}$ ). It is seen that the designed planar array has a compact size, high gain, sufficient efficiency, and beam-steering properties.

In addition, the characteristics of the $26 \mathrm{GHz}$ planar Quasi-Yagi antenna array with different numbers of the radiators $(2 \times 2,4 \times 4$, and $8 \times 8)$ are studied in Figure 20. As shown in Figure 20a, 
the designed planar arrays exhibit directional radiation beams with high gain levels. Figure 20b,c illustrates the reflection coefficient and mutual coupling $\left(S_{n n}\right.$ and $\left.S_{n m}\right)$ characteristics of the middle elements for the planar arrays. As shown, similar performances of the $S$ parameters with high isolation and low mutual coupling have been obtained for them. Reflection coefficients $\left(\mathrm{S}_{\mathrm{nn}}\right)$ of $-35,-48$, and $-43 \mathrm{~dB}$ have been achieved for $2 \times 2,4 \times 4$, and $8 \times 8$ planar arrays. Furthermore, the designed Quasi-Yagi antenna arrays provide a low mutual coupling characteristic (less than $-13 \mathrm{~dB}$ at $26 \mathrm{GHz}$ ).
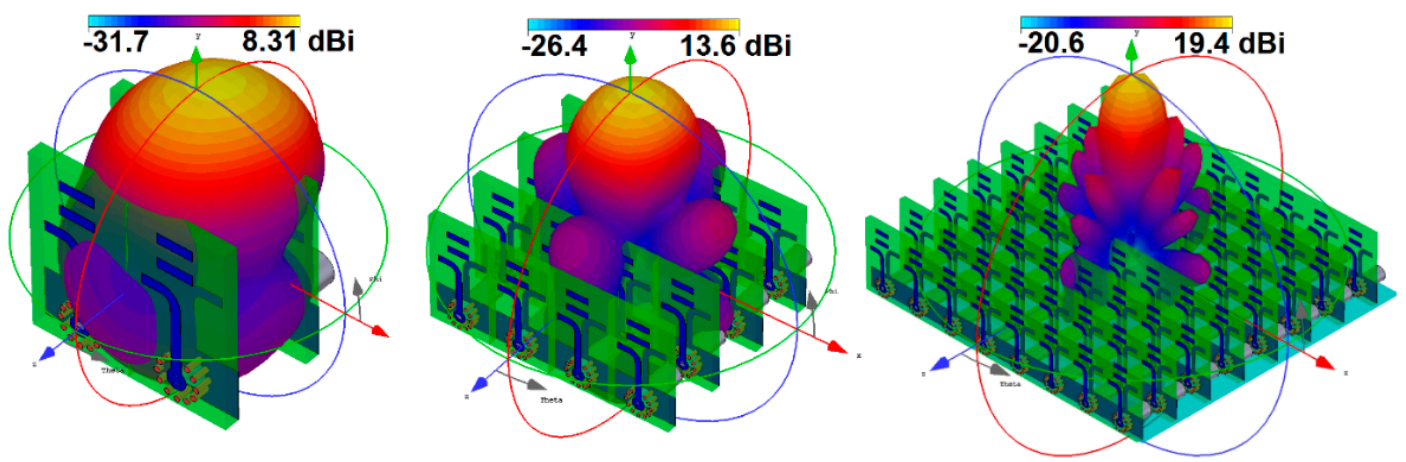

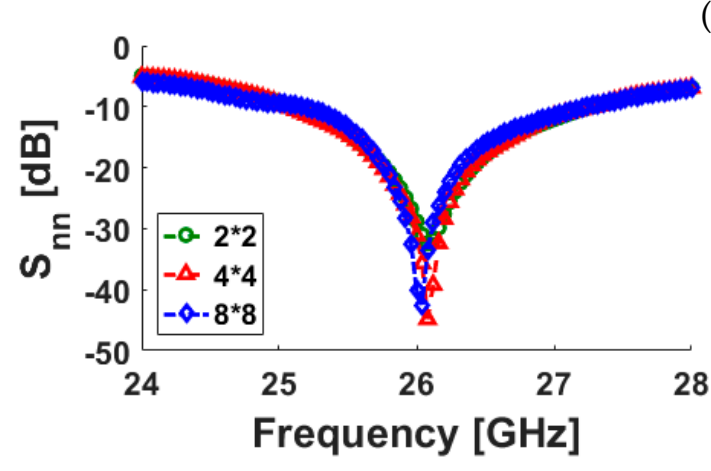

(b)

(a)

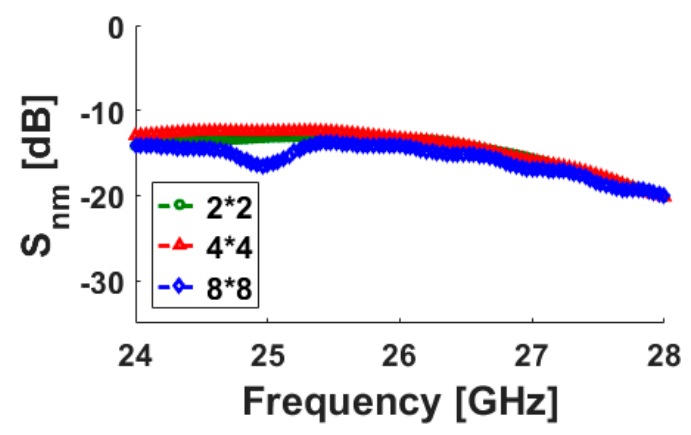

(c)

Figure 20. (a) Radiation beams, (b) $S_{n n}$, and (c) $S_{n m}$ characteristics of the planar arrays.

\section{Conclusions}

A phased array 5G smartphone antenna with Quasi-Yagi radiators was designed and investigated in this manuscript. The antenna array has well-defined/wide-scan radiation beams with end-fire modes at the $26 \mathrm{GHz} 5 \mathrm{G}$ band. Fundamental characteristics of the presented smartphone antenna array and its elements (Quasi-Yagi radiators) have been studied. SAR and user-impact on the 5G smartphone were also investigated. In addition, using the Quasi-Yagi antenna elements, $2 \times 2,4 \times 4$, and $8 \times 8$ planar phased array designs were designed, and their characteristics have been investigated. Based on the obtained result, the Quasi-Yagi antenna element, its linear and planar phased arrays, is promising for use in $5 \mathrm{G}$ cellular communications.

Author Contributions: Writing-original draft preparation, N.O.P., M.A., H.J.B., R.A.A.-A., J.R., and E.L.; writing-review and editing, N.O.P., H.J.B., J.R., and R.A.A.-A.; investigation, N.O.P., M.A., and H.J.B.; resources, N.O.P., J.R., and R.A.A.-A.; For other cases, all authors have participated.

Funding: This project has received funding from the European Union's Horizon 2020 Research and Innovation Programme under grant agreement H2020-MSCA-ITN-2016 SECRET-722424.

Acknowledgments: Authors wish to express their thanks for the support provided by the innovation programme under grant agreement H2020-MSCA-ITN-2016 SECRET-722424.

Conflicts of Interest: The authors declare no conflict of interest. 


\section{References}

1. Rappaport, T.S.; Sun, S.; Mayzus, R.; Zhao, H.; Azar, Y.; Wang, K.; Wong, G.N.; Schulz, J.K.; Samimi, M.; Gutierrez, F. Millimeter wave mobile communications for 5G cellular: It will work! IEEE Access 2013, 1, 335-349. [CrossRef]

2. Osseiran, A.; Boccardi, F.; Braun, V.; Kusume, K.; Marsch, P.; Maternia, M.; Queseth, O.; Schellmann, M.; Schotten, H.; Taoka, H.; et al. Scenarios for $5 \mathrm{G}$ mobile and wireless communications: The vision of the METIS project. IEEE Commun. Mag. 2014, 52, 26-35. [CrossRef]

3. Roh, W.; Seol, J.; Park, J.; Lee, B.; Lee, J.; Kim, Y.; Cho, J.; Cheun, K.; Aryanfar, F. Millimeter-wave beamforming as an enabling technology for $5 \mathrm{G}$ cellular communications: Theoretical feasibility and prototype results. IEEE Commun. Mag. 2014, 52, 106-113. [CrossRef]

4. Ofcom. Available online: https://www.ofcom.org.uk/ (accessed on 8 February 2017).

5. Bai, T.; Heath, R. Coverage and rate analysis for millimeter wave cellular networks. IEEE Trans. Wirel. Commun. 2015, 14, 1110-1114. [CrossRef]

6. Jilani, S.F.; Munoz, M.O.; Abbasi, Q.H.; Alomainy, A. Millimeter-wave liquid crystal polymer based conformal antenna array for 5G applications. IEEE Antennas Wirel. Propag. Lett. 2019, 18, 84-88. [CrossRef]

7. Ojaroudiparchin, N.; Shen, M.; Zhang, S.; Pedersen, G.F. A switchable 3-D-coverage-phased array antenna package for 5G mobile terminals. IEEE Antennas Wirel. Propag. Lett. 2016, 15, 1747-1750. [CrossRef]

8. Zhang, J.; Zhang, S.; Lin, X.; Fan, Y.; Pedersen, G. 3D radiation pattern reconfigurable phased array for transmission angle sensing in 5G mobile communication. Sensors 2018, 18, 4204. [CrossRef] [PubMed]

9. Ojaroudiparchin, N.; Shen, M.; Pedersen, G.F. Multi-layer 5G mobile phone antenna for multi-user MIMO communications. In Proceedings of the 2015 23rd Telecommunications Forum Telfor (TELFOR), Belgrade, Serbia, 24-26 November 2015.

10. Ojaroudiparchin, N.; Shen, M.; Pedersen, G.F. Beam-steerable microstrip-fed bow-tie antenna array for fifth generation cellular communications. In Proceedings of the 2016 10th European Conference on Antennas and Propagation (EuCAP), Davos, Switzerland, 10-15 April 2016.

11. Parchin, N.O.; Shen, M.; Pedersen, G.F. Small-size tapered slot antenna (TSA) design for use in 5G phased array applications. Appl. Comput. Electromagn. Soc. (ACES) J. 2017, 3, 1054-4887.

12. Xu, Q.; Biedka, M.; Wang, Y.E. Indented antenna arrays for high isolation: The growing interest in simultaneous-transmit-and-receive-based full-duplex communication systems. IEEE Antennas Propag. Mag. 2018, 60, 72-80. [CrossRef]

13. Morgan, M.; Weinreb, S. A millimeter-wave perpendicular coax-to-microstrip transition. In Proceedings of the 2002 IEEE MTT-S International Microwave Symposium Digest (Cat. No. 02CH37278), Seattle, WA, USA, 2-7 June 2002.

14. Zhao, A.; Ai, F. 5G mm-wave antenna array based on T-slot antenna for mobile terminals. In Proceedings of the 2018 IEEE Asia-Pacific Conference on Antennas and Propagation (APCAP), Auckland, New Zealand, 5-8 August 2018.

15. Ojaroudiparchin, N.; Shen, M.; Pedersen, G.F. A 28 GHz FR-4 compatible phased array antenna for 5G mobile phone applications. In Proceedings of the International Symposium on Antennas and Propagation (ISAP 2015), Tasmania, Australia, 9-12 November 2015.

16. Ojaroudi Parchin, N.; Al-Yasir, Y.; Abdulkhaleq, A.M.; Elfergani, I.; Rayit, A.; Noras, J.M.; Rodriguez, J.; Abd-Alhameed, R.A. Frequency reconfigurable antenna array for mm-Wave 5G mobile handsets. In Proceedings of the 9th International Conference on Broadband Communications, Networks, and Systems, Faro, Portugal, 19-20 September 2018.

17. Tatomirescu, A.; Oprian, A.; Zhekov, S.; Pedersen, G.F. Beam-steering array for handheld devices targeting 5G. In Proceedings of the 2015 International Symposium on Antennas and Propagation (ISAP), Hobart, TAS, Australia, 9-12 November 2015.

18. CST Microwave Studio; ver. 2017; CST: Framingham, MA, USA, 2017.

19. Kaneda, N.; Deal, W.R.; Qian, Y.; Waterhouse, R.; Itoh, T. A broadband planar quasi-Yagi antenna. IEEE Trans. Antennas Propag. 2002, 50, 1158-1160. [CrossRef]

20. Ansoft High Frequency Structure Simulation (HFSS); Ver. 17; Ansoft Corporation: Pittsburgh, PA, USA, 2017. 
21. Ojaroudiparchin, N.; Shen, M.; Pedersen, G.F. Investigation on the performance of low-profile insensitive antenna with improved radiation characteristics for the future 5G applications. Microw. Opt. Technol. Lett. 2016, 58, 2148-2158. [CrossRef]

22. Jamesn, J.R.; Hall, P.S. Handbook of Microstrip Antennas; Peter Peregrinus Ltd.: London, UK, 1989.

23. Amitay, N.; Galindo, V.; Wu, C.P. Theory and Analysis of Phased Array Antennas; Wiley Interscience: Hoboken, NJ, USA, 1972.

24. Hong, W.; Ko, S.T.; Lee, Y.; Baek, K.H. Multi-polarized antenna array configuration for mm-Wave 5G mobile terminals. In Proceedings of the 2015 International Workshop on Antenna Technology (iWAT), Seoul, Korea, 4-6 March 2015.

25. Ojaroudiparchin, N.; Shen, M.; Pedersen, G.F. $8 \times 8$ planar phased array antenna with high efficiency and insensitivity properties for 5G mobile base stations. In Proceedings of the 2016 10th European Conference on Antennas and Propagation (EuCAP), Davos, Switzerland, 10-15 April 2016.

26. Hansen, R.C. Phased Array Antennas; John Wiley \& Sons, Inc.: New York, NY, USA, 2009.

27. Parchin, N.O. Low-profile air-filled antenna for next generation wireless systems. Wirel. Pers. Commun. 2017, 97, 3293-3300. [CrossRef]

28. Khan, R.; Al-Hadi, A.A.; Soh, P.J. Recent advancements in user effect mitigation for mobile terminal antennas: A review. IEEE Trans. Electromagn. Compat. 2019, 16, 279-287. [CrossRef]

29. Bonev, I.B.; Franek, O.; Pedersen, G.F. Impact of the hand on the specific absorption rate in the head. Appl. Comput. Electromagn. Soc. (ACES) J. 2014, 29, 470-477.

30. Ojaroudiparchin, N.; Shen, M.; Pedersen, G.F. Design of Vivaldi antenna array with end-fire beam steering function for 5G mobile terminals. In Proceedings of the 2015 23rd Telecommunications Forum Telfor (TELFOR), Belgrade, Serbia, 24-26 November 2015.

31. Moustafa, J.; McEwan, N.J.; Abd-Alhameed, R.A.; Excell, P.S. Low SAR phased antenna array for mobile handsets. Appl. Comput. Electromagn. Soc. (ACES) J. 2006, 21, 196-205.

32. Faruque, M.R.I. Evaluation of EM absorption in human head with metamaterial attachment. Appl. Comput. Electromagn. Soc. (ACES) J. 2010, 25, 1097-1107.

33. Ojaroudiparchin, N.; Shen, M.; Pedersen, G.F. Low-cost planar mm-Wave phased array antenna for use in mobile satellite (MSAT) platforms. In Proceedings of the 23rd Telecommunications forum (TELFOR), Belgrade, Serbia, 24-26 November 2015; pp. 587-590.

34. Ojaroudi Parchin, N.; Abd-Alhameed, R.A. A compact Vivaldi antenna array for 5G channel sounding applications. In Proceedings of the 12th European Conference on Antennas and Propagation (EuCAP 2018), London, UK, 9-13 April 2018. 